\title{
A functional genomics approach to identify pathways of drug resistance in medulloblastoma
}

\author{
Kelsey C. Bertrand ${ }^{1,2}$, Claudia C. Faria ${ }^{3}$, Patryk Skowron 4,5,6,7, Amanda Luck ${ }^{4,5,6,7}$, Livia Garzia 4,5,6,7, \\ Xiaochong Wu ${ }^{4,5,6,7}$, Sameer Agnihotri ${ }^{8}$, Christian A. Smith ${ }^{4,5,6,7}$, Michael D. Taylor ${ }^{4,5,6,7}$, Stephen C. Mack ${ }^{1,2^{*}}$ (D) and \\ James T. Rutka ${ }^{4,5,6,7^{*}}$
}

Keywords: Cancer, Brain tumor, Medulloblastoma, Drug resistance, Functional genomics, Transposon mutagenesis, Sleeping beauty

Medulloblastoma (MB) is the most common malignant pediatric brain tumor [10]. Although continued advances have been made in our understanding of medulloblastoma, questions remain about its etiology and treatment $[3,4,7,9,13-15,17,18]$. Medulloblastoma is classified into four demographically, clinically, and molecularly distinct subgroups called WNT, SHH, Group 3 and Group 4 (As reviewed in [19]), and further divided into additional distinct molecular subtypes [3, 14]. While patients with (WNT) pathway driven medulloblastoma have favorable outcomes, recurrence rates are higher in other subtypes such as $\mathrm{SHH}$, which tend to recur locally, and Group 3 and 4, which are associated with distal metastases [16]. The biology of metastatic medulloblastoma is distinct from primary medulloblastoma; representing potentially a different therapeutic disease $[6,8,13]$. Approaches are needed to identify pathways of therapy resistance in both primary and metastatic compartments of medulloblastoma to guide selection of targeted therapies. As an example, the MET proto-oncogene is upregulated in $\mathrm{SHH}$ and Group 3 medulloblastoma, and can be targeted by treatment of cells and mouse models with the small molecule inhibitor, Foretinib [4]. We utilized a spontaneous metastatic mouse model of medulloblastoma driven by the Sleeping Beauty mutagenesis transposon system [21] to pinpoint functional drivers

\footnotetext{
* Correspondence: stephen.mack@bcm.edu; james.rutka@sickkids.ca ${ }^{1}$ Department of Pediatrics, Division of Hematology and Oncology, Baylor College of Medicine, Houston, USA

${ }^{4}$ Department of Developmental and Stem Cell Biology and Regenerative Medicine, The Hospital for Sick Children, Toronto, Canada

Full list of author information is available at the end of the article
}

and pathways of resistance to Foretinib [4] in both primary and metastatic medulloblastoma. This serves as a novel approach to dissecting patterns of therapy resistance at multiple tumor sites simultaneously which can be readily applied to other cancer systems.

\section{Approach}

A medulloblastoma sleeping beauty transposon mutagenesis mouse model (Ptch $+/-$, SB100/SB68, T2Onc), that spontaneously develops primary and metastatic medulloblastoma with $100 \%$ penetrance, was used to identify pathways of Foretinib resistance [13] (Fig. 1a). This system allows for entrapment of both oncogenes and tumor suppressor genes, which can be identified by next-generation sequencing of transposon insertion sites [21]. We showed previously that Foretinib is an effective inhibitor of the MET pathway in $\mathrm{SHH}$ and Group 3 medulloblastoma mouse models [4] (Fig. 1b). Following tumor establishment in a time frame of 30-35 days, mice were treated with vehicle or Foretinib, through continuous osmotic pump infusion into the cerebrospinal fluid for 28 days at a rate of $0.25 \mu \mathrm{l}$ per hour (Fig. 1a). Resistant primary and metastatic tumors were harvested and genomic common insertion sites (gCIS) were identified by SPLINK PCR combined with paired-end Illumina high-throughput sequencing (Fig. 1a), to pinpoint genetic drivers of therapy resistance.

\section{Results and discussion}

We leveraged the Sleeping Beauty transposon system to identify pathways of resistance to Foretinib as a proofof-concept strategy, which could be applied to other 


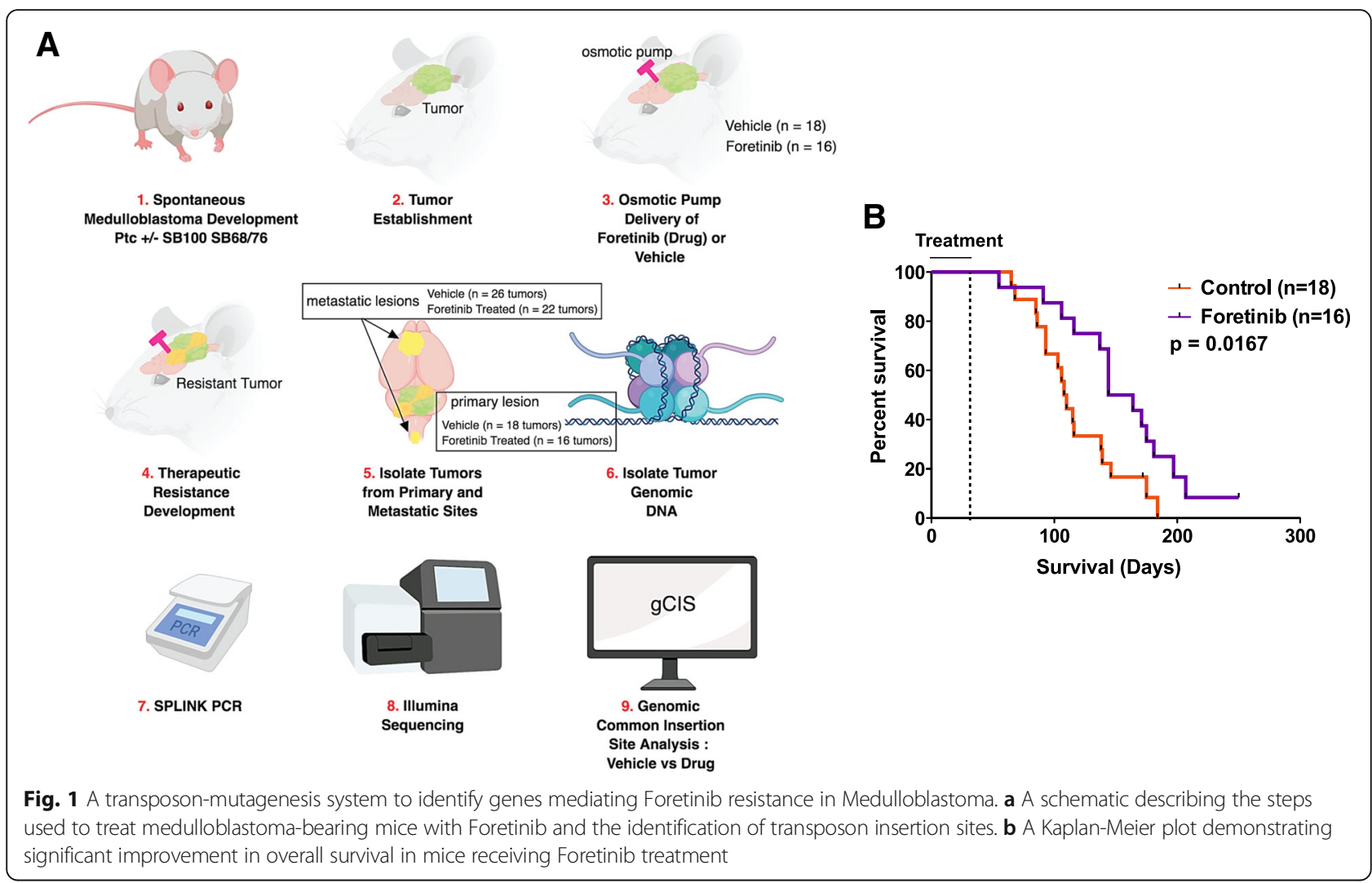

cancer models and systems. In primary medulloblastoma, the patterns of gCISs in Foretinib-treated mice were highly distinct from control mice, thus supporting that the underlying mechanisms of primary medulloblastoma are different from tumors receiving Foretinib therapy (Fig. 2a). In primary Foretinib-treated tumors, we identified specific insertions in known tumor oncogenes and tumor suppressor genes such as Pten, Cdh2, Egfr, and Acvr1b [1, 5, 20] (Fig. 2a-c). As two examples, PTEN insertions were predicted to being inactivating mutations by their location and antisense orientation within the $5^{\prime}$ end of the gene introducing an early poly A transcriptional termination signal. $\mathrm{CDH} 2$ insertions were predicted to either promote expression of small isoforms of $\mathrm{CDH} 2$ and/or ablate expression of long isoforms of the gene. The majority of these candidates have been shown to be mutated in cancer when compared against the Catalogue of Somatic Mutations in Cancer database and several have been previously implicated in brain tumorigenesis [1, 2, 5, 20] (Fig. 2b). Further, the mechanisms mediating Foretinib-resistance in primary site tumors were over-represented by pathways involved in protein metabolism, specifically ubiquitin-mediated protein degradation (Fig. 2d). Our findings demonstrate that mice bearing medulloblastoma and receiving Foretinib therapy exhibit distinct pathway alterations from primary lesions. These pathways, identified through Sleeping Beauty transposon insertion analysis, represent candidate drivers and potential targets in Foretinib resistant medulloblastoma.

Historically, metastatic disease has been assumed to be highly similar to primary tumors, and therefore presumably equally responsive to treatments designed to target primary lesions. Using the Sleeping Beauty Transposon system, we show that primary and metastatic medulloblastoma exhibit distinct patterns of genetic alterations (Fig. 3a, Additional file 1: Table S1-S4). gCISs identified in primary medulloblastoma included transcriptional regulators such as Crebbp, and Ep300, and in metastatic medulloblastoma immune response-related genes such as C6, A2m, and Pkp2 (Additional file 1: Table S5-S8). These data support that the primary and metastatic compartments of medulloblastoma are driven by distinct molecular mechanisms [12]. We next asked whether metastatic medulloblastoma might evolve different or convergent pathways of resistance, as compared to the primary-treated tumors. We found that metastatic medulloblastoma receiving Foretinib therapy exhibited distinct patterns of genomic insertions compared to the metastatic compartment of vehicle treated mice (Fig. 3b). Furthermore, metastatic gCISs were highly divergent from the primary compartment in mice, which had also received Foretinib therapy (Fig. 3c). Foretinib-resistant metastatic medulloblastoma insertions included Basp1, Flt4, Mllt10, and Asxl2 (Fig. 3d,e) and pathways involved in cellular 


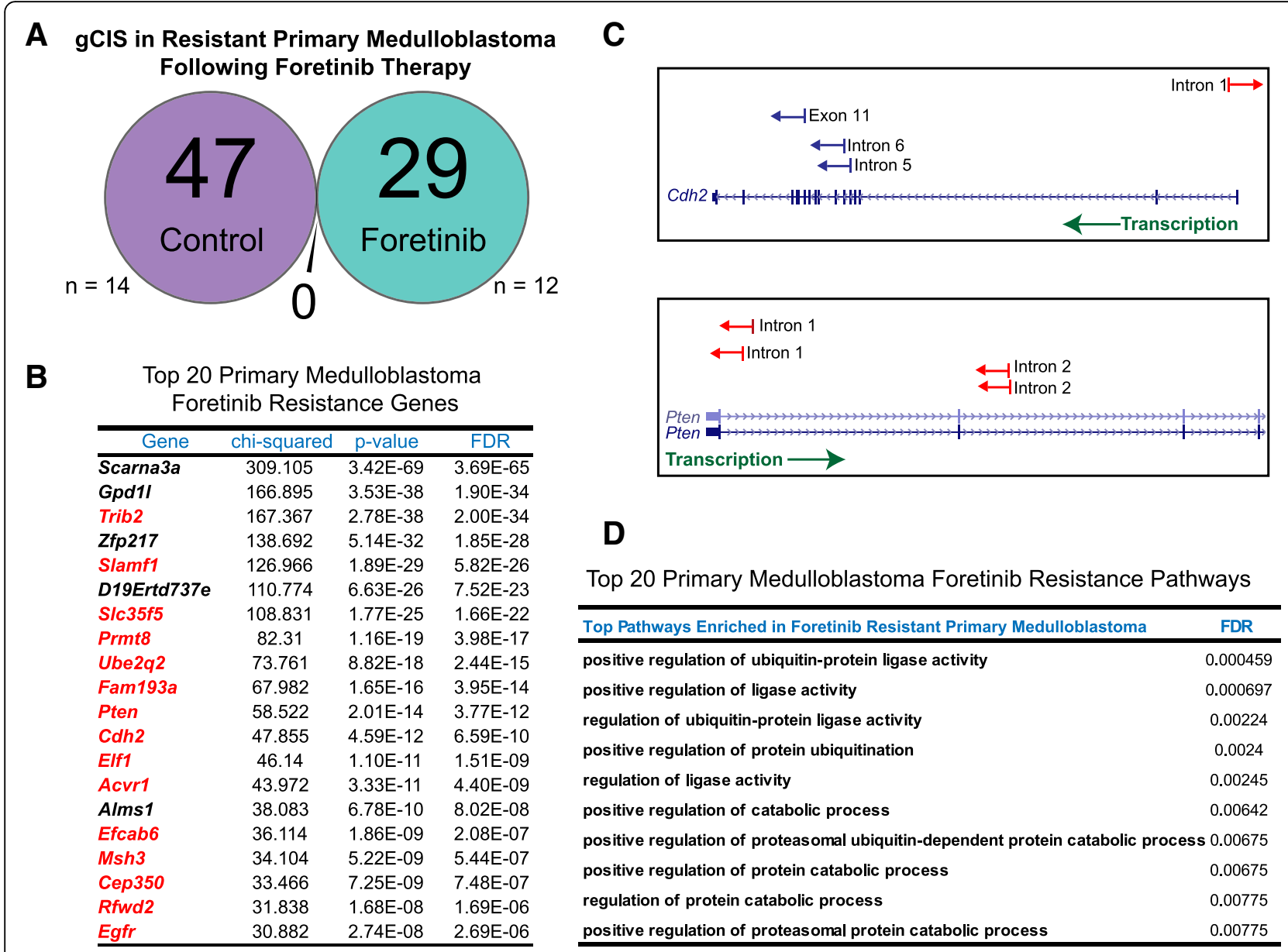

Fig. 2 Transposon insertion patterns are divergent in primary medulloblastoma receiving Foretinib therapy. a A Venn diagram illustrating the number of statistically significant gCISs exclusive or shared gCISs between vehicle $(n=14)$ and Foretinib $(n=12)$ treated primary medulloblastoma. $\mathbf{b}$ A table showing the Top 20 statistically significant Foretinib resistance genes in primary medulloblastoma. Highlighted in red are genes which have been reported to be mutated in cancer when compared against the COSMIC database. $\mathbf{c}$ Examples of transposon insertions in Cdh2 and Pten and their direction of orientation (red = anti-sense, blue $=$ sense) relative to direction transcription (green). $\mathbf{d}$ Pathway analysis of Foretinib-resistance genes in primary medulloblastoma identified using GeneMania

metabolism (Fig. 3f). These findings demonstrate that primary and metastatic medulloblastoma are molecularly distinct; hence their response and resistance to therapy may be highly divergent. Furthermore we demonstrate the effectiveness of functional genomic mapping to simultaneously identify putative drivers of tumorigenesis in distinct tumor compartments.

\section{Conclusion}

Our study has identified potential pathways that medulloblastoma cells may co-opt to overcome Foretinib inhibition, and provides a strategy for which drug resistance pathways to other medulloblastoma targeted therapies may be identified. Prospective identification of these pathways could be used to determine combinatory treatments that may be effective for resistant primary and metastatic tumor clones. We further demonstrate in our model that primary and metastatic medulloblastoma are genetically distinct, and in response to Foretinib-therapy, exhibit divergent mechanisms of resistance. A limitation of our method is that while driver pathways may be identified, they may not represent the exact genes targeted in resistant human primary tumors. Therefore, integrative functional mouse modeling using this Sleeping Beauty Approach paired with genomic characterization of resistant primary tumors, may prioritize pathways and specific targets that mediate cancer therapy resistance. Finally, our data lends support that treatments armed against genetic targets in the primary site may be ineffective for metastatic lesions, and that potentially distinct genetic evolution occurs between primary and metastatic medulloblastoma under therapy.

\section{Materials and methods}

Animal studies

All mouse studies were approved and performed in accordance to the policies and regulations of the Institutional 


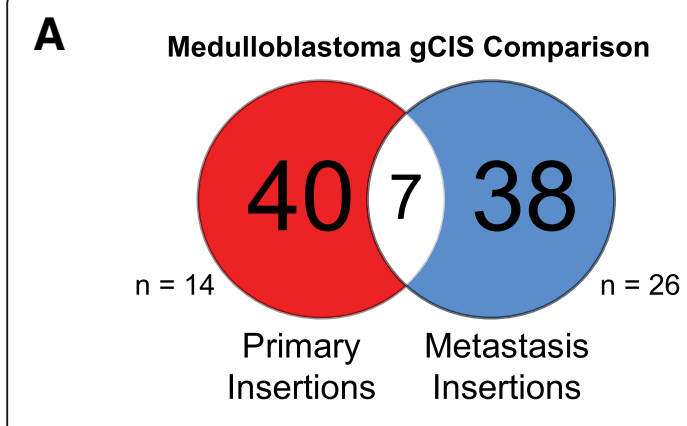

B

gClS in Resistant Metastatic Medulloblastoma Following Foretinib Therapy

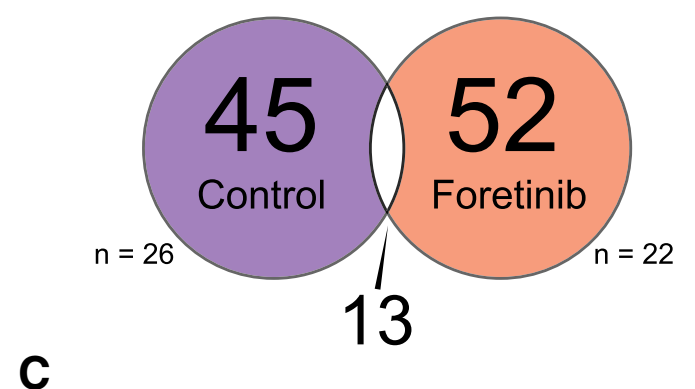

gCISs in Primary versus Metastatic Medulloblastoma Following Foretinib Therapy

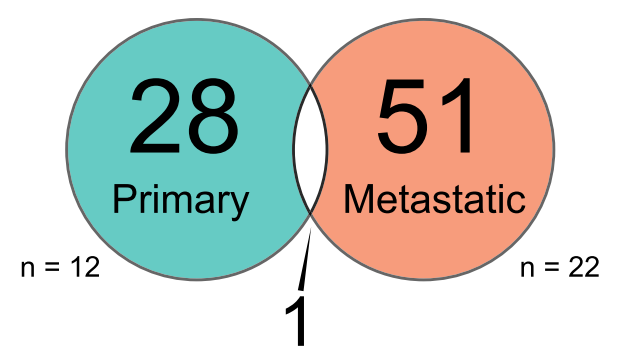

D
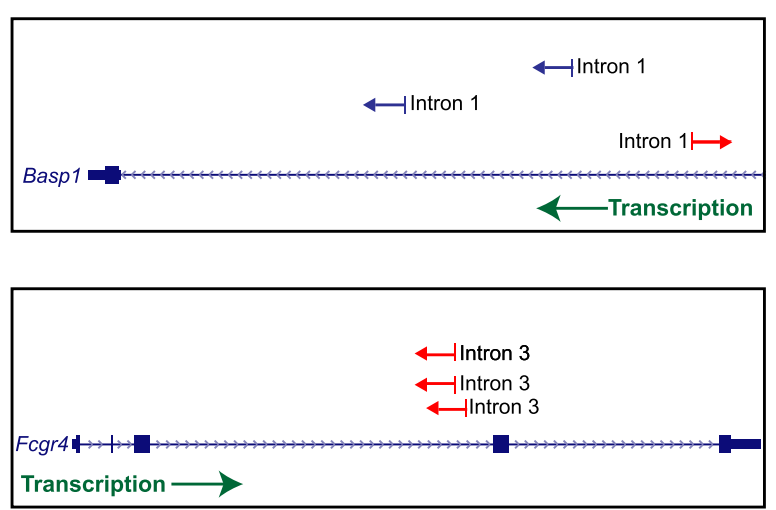

E

Top 20 Metastatic Medulloblastoma

Foretinib Resistance Genes

\begin{tabular}{lccc}
\hline \multicolumn{1}{c}{ Gene } & chi-squared & p-value & FDR \\
\hline Basp1 & 200.842 & $1.37 \mathrm{E}-45$ & $7.37 \mathrm{E}-42$ \\
1700009P17Rik & 170.963 & $4.56 \mathrm{E}-39$ & $1.96 \mathrm{E}-35$ \\
Acot10 & 144.151 & $3.29 \mathrm{E}-33$ & $1.18 \mathrm{E}-29$ \\
Fcgr4 & 130.054 & $3.99 \mathrm{E}-30$ & $1.23 \mathrm{E}-26$ \\
Rai14 & 123.002 & $1.39 \mathrm{E}-28$ & $3.75 \mathrm{E}-25$ \\
Fastkd5 & 122.387 & $1.90 \mathrm{E}-28$ & $4.55 \mathrm{E}-25$ \\
1110020G09Rik & 118.949 & $1.07 \mathrm{E}-27$ & $2.31 \mathrm{E}-24$ \\
Psmg4 & 114.966 & $8.00 \mathrm{E}-27$ & $1.57 \mathrm{E}-23$ \\
Rnasen & 100.815 & $1.01 \mathrm{E}-23$ & $1.67 \mathrm{E}-20$ \\
Fundc1 & 84.92 & $3.11 \mathrm{E}-20$ & $4.18 \mathrm{E}-17$ \\
Tnfsf4 & 83.443 & $6.56 \mathrm{E}-20$ & $8.31 \mathrm{E}-17$ \\
Dtx2 & 80.086 & $3.58 \mathrm{E}-19$ & $4.06 \mathrm{E}-16$ \\
Colec10 & 79.427 & $5.00 \mathrm{E}-19$ & $5.39 \mathrm{E}-16$ \\
Flt4 & 71.786 & $2.40 \mathrm{E}-17$ & $2.07 \mathrm{E}-14$ \\
Ubox5 & 66.374 & $3.73 \mathrm{E}-16$ & $2.77 \mathrm{E}-13$ \\
Fyb & 65.863 & $4.83 \mathrm{E}-16$ & $3.47 \mathrm{E}-13$ \\
Ttc33 & 58.195 & $2.37 \mathrm{E}-14$ & $1.28 \mathrm{E}-11$ \\
Trps1 & 53.156 & $3.08 \mathrm{E}-13$ & $1.28 \mathrm{E}-10$ \\
Dcaf13 & 51.427 & $7.43 \mathrm{E}-13$ & $2.91 \mathrm{E}-10$ \\
Laptm4b & 50.96 & $9.42 \mathrm{E}-13$ & $3.56 \mathrm{E}-10$ \\
\hline
\end{tabular}

$\mathbf{F}$

Top 20 Metastatic Medulloblastoma Foretinib Resistance Pathways

\begin{tabular}{lc}
\hline Top Pathw ays Enriched in Foretinib Resistant Metastatic Medulloblastoma & FDR \\
\hline Cellular respiration & 0.0223 \\
Energy derivation by oxidation of organic compounds & 0.0223 \\
Generation of precursor metabolites and energy & 0.0749 \\
Transferase activity, transferring nitrogenous groups & 0.0749 \\
Transaminase activity & 0.0749 \\
\hline
\end{tabular}

Fig. 3 Divergent patterns of transposon insertions in metastatic medulloblastoma following Foretinib therapy. a A Venn diagram illustrating the number of statistically significant gCISs identified as exclusive or shared between primary $(n=14)$ and metastatic medulloblastoma $(n=26)$. $\mathbf{b} \mathrm{A}$ Venn diagram illustrating the number of statistically significant gCISs exclusive or shared gCISs between vehicle $(n=26)$ and Foretinib treated metastatic medulloblastoma $(n=22)$. c A Venn diagram comparing the gCISs between primary $(n=12)$ and metastatic $(n=22)$ Foretinib treated medulloblastoma. d A table showing the Top 20 statistically significant Foretinib resistance genes in metastatic medulloblastoma. Highlighted in red are genes, which have been reported to be mutated in cancer when compared against the COSMIC database. e Examples of transposon insertions in Baspl and Fcgr4 and their direction of orientation (red = anti-sense, blue = sense) relative to direction transcription (green). $\mathbf{f}$ Pathway analysis of Foretinib-resistance genes in metastatic medulloblastoma identified using GeneMania

Animal Care and Use Committee of the University of Toronto and the Hospital for Sick Children. A medulloblastoma Sleeping Beauty transposon mutagenesis murine model $\left(\right.$ Ptch $^{+/} /$SB11/T2Onc) was used, which frequently and spontaneously develops primary and metastatic MB.

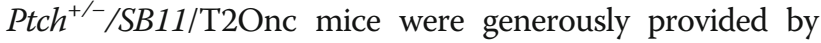

Dr. Michael D. Taylor, Hospital for Sick Children, Toronto, Canada. Mice at post-natal day 30-35 were treated with vehicle or Foretinib $(6 \mathrm{mg} / \mathrm{kg})$, via Alzet osmotic pump (Model 2004) slow-infusion into the cerebrospinal fluid of the right lateral ventricle, for 28 days at a rate of $0.25 \mathrm{ul} /$ hour. Nucleic acid extractions were carried out as 
previously described. Statistical differences in survival curves of mice was assessed using a Kaplan-Meier estimate and log-rank test.

\section{Splinkerette PCR and common genomic insertion site analysis}

Transposon common insertion sites were identified by SPLINK PCR of tumour DNA, followed by 100 bp pairedend Illumina next-generation sequencing (HiSeq 2500). Genomic DNA was digested and ligated with linker +/primers, amplified through PCR, then further amplified with barcoded primers through a second PCR. DNA was then purified and prepared for sequencing; protocol as previously described [10]. Gene pathways were identified by querying gene lists with GeneMania [11], and significance measured using a hypergeometric distribution test. Data availability: The datasets supporting the conclusions of this article are included within this article. Raw data will be made openly available through the GEO repository.

\section{Additional file}

Additional file 1: Table S1. Control Insertions - Primary Medulloblastoma. Table S2. Control Insertions - Primary Medulloblastoma (per sample). Table S3. Foretinib Specific Insertions - Primary Medulloblastoma. Table S4. Foretinib Insertions - Primary Medulloblastoma (per sample). Table S5. Control Insertions - Metastatic Medulloblastoma. Table S6. Control Insertions - Metastatic Medulloblastoma (per sample). Table S7. Foretinib Specific Insertions - Metastatic Medulloblastoma. Table S8. Foretinib Insertions - Metastatic Medulloblastoma (per sample). (XLSX 479 kb)

\section{Acknowledgements}

We would like to thank Rob Denroche for assistance in DNA sequence analysis. This study was supported by the Canadian Cancer Society Research Institute (grant \#2011-70051), the Pediatric Brain Tumor Formation of the United States, the Brain Tumour Foundation of Canada, b.r.a.i.n.child, Meagan's Walk, and the Wiley Fund at The Hospital for Sick Children. SCM is supported by an Alex's Lemonade Stand Young Investigator Award, The CIHR Banting Fellowship, The Cancer Prevention Research Institute of Texas (SCM-RR170023), Rally research grant, BEAR Necessities Pediatric Cancer Foundation Grant, Children's Cancer Research Fund award, and Baylor College of Medicine Junior Faculty Award, Children's Brain Tumor Foundation Grant, and an Alex's Lemonade Stand Foundation Young Investigator and A award. MDT is supported by The Garron Family Chair in Childhood Cancer Research, and grants from the Pediatric Brain Tumour Foundation, Grand Challenge Award from CureSearch for Children's Cancer, the National Institutes of Health (R01CA148699 R01CA159859), The Terry Fox Research Institute, and Brainchild. MDT is also supported by a Stand Up To Cancer St. Baldrick's Pediatric Dream Team Translational Research Grant (SU2CAACR-DT1113). Stand Up To Cancer is a program of the Entertainment Industry Foundation administered by the American Association for Cancer Research.

\section{Authors' contributions}

KCB led the study, interpreted the genomic analysis, and wrote the manuscript. CCF performed the animal experiments and collected tissue for the study. PS and LG performed the Sleeping Beauty Insertion analysis. AL assisted with pump implantation, and tissue collection and organization. XW provided the transgenic mice. SA, JNR, CAS, SCM, MDT provided analytical advice, data interpretation, and assisted with manuscript preparation. JTR funded and directed the entire research effort, from data interpretation, analysis, to manuscript preparation. All authors read and approved the final manuscript.

\section{Competing interests}

The authors declare that they have no competing interests.

\section{Publisher's Note}

Springer Nature remains neutral with regard to jurisdictional claims in published maps and institutional affiliations.

\section{Author details}

${ }^{1}$ Department of Pediatrics, Division of Hematology and Oncology, Baylor College of Medicine, Houston, USA. ${ }^{2}$ Brain Tumor Program, Cancer and Hematology Centers, Texas Children's Hospital, Houston, USA. ${ }^{3}$ Department of Neurosurgery, Hospital de Santa Maria, Centro Hospitalar Lisboa Norte, EPE, Lisbon, Portugal. ${ }^{4}$ Department of Developmental and Stem Cell Biology and Regenerative Medicine, The Hospital for Sick Children, Toronto, Canada. ${ }^{5}$ Arthur and Sonia Labatt Brain Tumor Research Center, The Hospital for Sick Children, Toronto, Canada. ${ }^{6}$ Department of Laboratory Medicine and Pathobiology, University of Toronto, Toronto, Canada. ${ }^{7}$ Department of Neurosurgery, The Hospital for Sick Children, Toronto, Canada. ${ }^{8}$ Department of Neurological Surgery, Children's Hospital, University of Pittsburgh School of Medicine, Pittsburgh, USA.

Received: 9 November 2018 Accepted: 19 December 2018 Published online: 27 December 2018

\section{References}

1. Brennan CW et al (2013) The somatic genomic landscape of glioblastoma. Cell 155:462-477. https://doi.org/10.1016/j.cell.2013.09.034

2. Buczkowicz P et al (2014) Genomic analysis of diffuse intrinsic pontine gliomas identifies three molecular subgroups and recurrent activating ACVR1 mutations. Nat Gen 46:451-456. https://doi.org/10.1038/ng.2936

3. Cavalli FMG et al (2017) Intertumoral Heterogeneity within Medulloblastoma Subgroups. Cancer cell 31:737-754.e736. https://doi.org/10.1016/j.ccell.2017.05.005

4. Faria CC et al (2015) Foretinib is effective therapy for metastatic sonic hedgehog medulloblastoma. Cancer Res 75:134-146. https://doi.org/10. 1158/0008-5472.can-13-3629

5. Fontebasso AM et al (2014) Recurrent somatic mutations in ACVR1 in pediatric midline high-grade astrocytoma. 46:462-466. https://doi.org/10. 1038/ng.2950

6. Genovesi LA et al (2013) Sleeping Beauty mutagenesis in a mouse medulloblastoma model defines networks that discriminate between human molecular subgroups. Proc Natl Acad Sci U S A 110:E4325-E4334. https://doi.org/10.1073/pnas.1318639110

7. Hovestadt $V$ et al (2014) Decoding the regulatory landscape of medulloblastoma using DNA methylation sequencing. Nature 510:537-541. https://doi.org/10.1038/nature13268

8. Jenkins NC et al (2014) Genetic drivers of metastatic dissemination in sonic hedgehog medulloblastoma. Acta Neuropathologica Commun 2:85. https:// doi.org/10.1186/preaccept-1860372034135162

9. Kool M et al (2014) Genome sequencing of SHH medulloblastoma predicts genotype-related response to smoothened inhibition. Cancer cell 25:393405. https://doi.org/10.1016/j.ccr.2014.02.004

10. Louis DN et al (2007) The 2007 WHO classification of tumours of the central nervous system. Acta neuropathologica 114:97-109. https://doi.org/10.1007/ s00401-007-0243-4

11. Montojo J, Zuberi K, Rodriguez H, Bader GD, Morris Q (2014) GeneMANIA: Fast gene network construction and function prediction for Cytoscape. F1000Res 3:153. https://doi.org/10.12688/f1000research.4572.1

12. Morrissy AS et al (2016) Divergent clonal selection dominates medulloblastoma at recurrence. Nature 529:351-357. https://doi.org/10. 1038/nature16478

13. Northcott PA et al (2012) Subgroup-specific structural variation across 1,000 medulloblastoma genomes. Nature 488:49-56. https://doi.org/10.1038/ nature11327

14. Northcott PA et al (2017) The whole-genome landscape of medulloblastoma subtypes. Nature 547:311-317. https://doi.org/10.1038/nature22973

15. Pugh TJ et al (2012) Medulloblastoma exome sequencing uncovers subtype-specific somatic mutations. Nature 488:106-110. https://doi.org/10. 1038/nature11329

16. Ramaswamy $V$ et al (2013) Recurrence patterns across medulloblastoma subgroups: an integrated clinical and molecular analysis. Lancet Oncol 14 1200-1207. https://doi.org/10.1016/s1470-2045(13)70449-2 
17. Robinson $\mathrm{G}$ et al (2012) Novel mutations target distinct subgroups of medulloblastoma. Nature 488:43-48. https://doi.org/10.1038/nature11213

18. Shih DJ et al (2014) Cytogenetic prognostication within medulloblastoma subgroups. J Clin Oncol 32:886-896. https://doi.org/10.1200/jco.2013.50.9539

19. Taylor MD et al (2012) Molecular subgroups of medulloblastoma: the current consensus. Acta Neuropathologica 123:465-472. https://doi.org/10. 1007/s00401-011-0922-z

20. Wu G et al (2014) The genomic landscape of diffuse intrinsic pontine glioma and pediatric non-brainstem high-grade glioma. Nat Gen 46:444450. https://doi.org/10.1038/ng.2938

21. Wu X et al (2012) Clonal selection drives genetic divergence of metastatic medulloblastoma. Nature 482:529-533. https://doi.org/10.1038/nature10825

Ready to submit your research? Choose BMC and benefit from:

- fast, convenient online submission

- thorough peer review by experienced researchers in your field

- rapid publication on acceptance

- support for research data, including large and complex data types

- gold Open Access which fosters wider collaboration and increased citations

- maximum visibility for your research: over $100 \mathrm{M}$ website views per year

At BMC, research is always in progress.

Learn more biomedcentral.com/submissions 